\title{
Spontaneous resolution of massive expulsive suprachoroidal hemorrhage with good long-term visual outcome: a case report
}

This article was published in the following Dove Press journal:

International Medical Case Reports Journal

9 September 2015

Number of times this article has been viewed

\section{Seung-Jun Lee* \\ Jang Hun Lee* \\ Sang Wook Park \\ Moosang Kim \\ Sang Beom Han}

Department of Ophthalmology,

Kangwon National University

Hospital, Kangwon National

University Graduate School of

Medicine, Chuncheon, Korea

*These authors contributed equally to this work
Correspondence: Sang Beom Han Department of Ophthalmology, Kangwon National University Hospital, Kangwon National University Graduate School of Medicine, 156 Baengnyeong-ro, Chuncheon, Gangwon 200-722, Korea $\mathrm{Tel}+82332589210$

Fax +82 332582296

Email m.sangbeom.han@gmail.com
Introduction: Massive suprachoroidal hemorrhage ( $\mathrm{SCH}$ ) that completely fills the vitreous cavity rarely resolves spontaneously. Even in cases with spontaneous resolution, long-term visual prognosis is disappointing due to retinal detachment or deterioration of preexisting ocular diseases. To the best of our knowledge, the first case of spontaneous resolution of massive $\mathrm{SCH}$ is reported with good long-term visual and anatomic outcome.

Case presentation: An 80-year-old Asian woman with high myopia underwent cataract surgery in the right eye. Posterior capsular rupture occurred during phacoemulsification. At the conclusion of scleral fixation of intraocular lens, expulsive SCH developed. After 1 day, best-corrected visual acuity (BCVA) was hand motion. Ultrasonography revealed massive SCH with central apposition. At 2nd week, decrease in $\mathrm{SCH}$ with resolution of central apposition was observed. At 6th week, BCVA improved to 20/120. Ultrasonography and fundus examination revealed complete resorption of the SCH and flat retina. At 1 year, BCVA was 20/50. Fundus was flat without any signs of retinal detachment.

Conclusion: Favorable anatomic and visual outcomes can be attained with conservative management even in cases of massive expulsive $\mathrm{SCH}$, particularly when central retinal apposition resolves in 2 weeks.

Keywords: conservative management, suprachoroidal hemorrhage, spontaneous resolution

\section{Introduction}

Suprachoroidal hemorrhage $(\mathrm{SCH})$ is a rare but potentially devastating complication of intraocular surgery. ${ }^{1}$ Although surgical intervention is often tried particularly in massive $\mathrm{SCH}$, visual outcome is disappointing. ${ }^{1-4}$ Although resolution of massive $\mathrm{SCH}$ with conservative management has been reported, long-term visual prognosis is poor. ${ }^{2}$ A case of spontaneous resolution of intraoperative massive $\mathrm{SCH}$ is recently examined with a good long-term visual outcome, thus herein report the case.

\section{Case presentation}

An 80-year-old Asian woman underwent cataract surgery in the right eye (OD). Preoperatively, best-corrected visual acuity (BCVA) was 20/70 OD with spherical equivalent of -6.50 diopters. Axial length measured with an optical biometer (IOLMaster, Carl Zeiss Meditec AG, Jena, Germany) was 26.08 mm OD. During phacoemulsification, posterior capsular rupture occurred. After aspiration of the lens fragments, anterior vitrectomy and scleral fixation of intraocular lens (IOL) was performed. Immediately after finishing the scleral fixation, a sudden elevation of dark-brown choroidal mass with rapid progression was detected. The main and side-port incisions 
were promptly closed with an intracameral instillation of $2 \%$ pilocarpine hydrochloride. A small amount of viscoelastic was left in the intracameral space to prevent vitreous incarceration. Intraocular pressure (IOP) was $37 \mathrm{mmHg}$ OD immediately after surgery. After administration of intravenous 15\% mannitol $200 \mathrm{~mL}$, IOP decreased to $22 \mathrm{mmHg}$. After 1 day, BCVA was hand motion (HM), and IOP was $15 \mathrm{mmHg}$ OD. Ultrasonography revealed $\mathrm{SCH}$ with central apposition (Figure 1A), and anterior segment examination showed a massive SCH touching the IOL (Figure 1B). Vitreous incarceration to wound was never found, although vitreous prolapse to intracameral space was observed. After 2 weeks, BCVA was HM, and IOP was $11 \mathrm{mmHg}$ OD. Ultrasonography showed decrease in $\mathrm{SCH}$ with resolution of central apposition. At postoperative 6 weeks, BCVA improved to 20/120 and IOP was $11 \mathrm{mmHg}$. Ultrasonography showed a complete resorption of the $\mathrm{SCH}$ (Figure 1C), and the fundus was flat (Figure 1D). At postoperative 1 year, BCVA improved to $20 / 50$ and IOP was $12 \mathrm{mmHg}$. Fundus was flat without any signs of retinal detachment.

\section{Consent}

Written informed consent was obtained from the patient for publication of this case report and any accompanying images.
Due to the retrospective nature and report of a single case, ethics approval was not requested. This study conformed to the tenets of the Declaration of Helsinki in respect of the testing of the device on humans. A copy of the written consent is available for review by the Editor of this journal.

\section{Discussion}

Current trend in the treatment of SCH includes early surgical intervention, particularly in cases of massive $\mathrm{SCH}$ with central retinal apposition, so-called "kissing configuration". 1 Meier and Wiedemann ${ }^{4}$ suggested that surgical treatment, including drainage by sclerotomy and vitrectomy, should be performed to minimize the damage caused by SCH. However, there exist evidences that central apposition may not be an absolute indication for surgery., ${ }^{2,3}$ Chu et $\mathrm{al}^{2}$ reported that the mean duration of central apposition in SCH was 15 days, and central choroidal elevation began to decrease rapidly at 3rd week, although peripheral elevation persisted for 6 weeks or longer. They also showed that all four cases with massive intraoperative $\mathrm{SCH}$ spontaneously resolved. ${ }^{2} \mathrm{Scott}$ et $\mathrm{al}^{3}$ demonstrated that no significant difference in visual outcome between observational management and surgical intervention in a study including 51 cases of massive $\mathrm{SCH}$. They reported that the duration of central apposition for
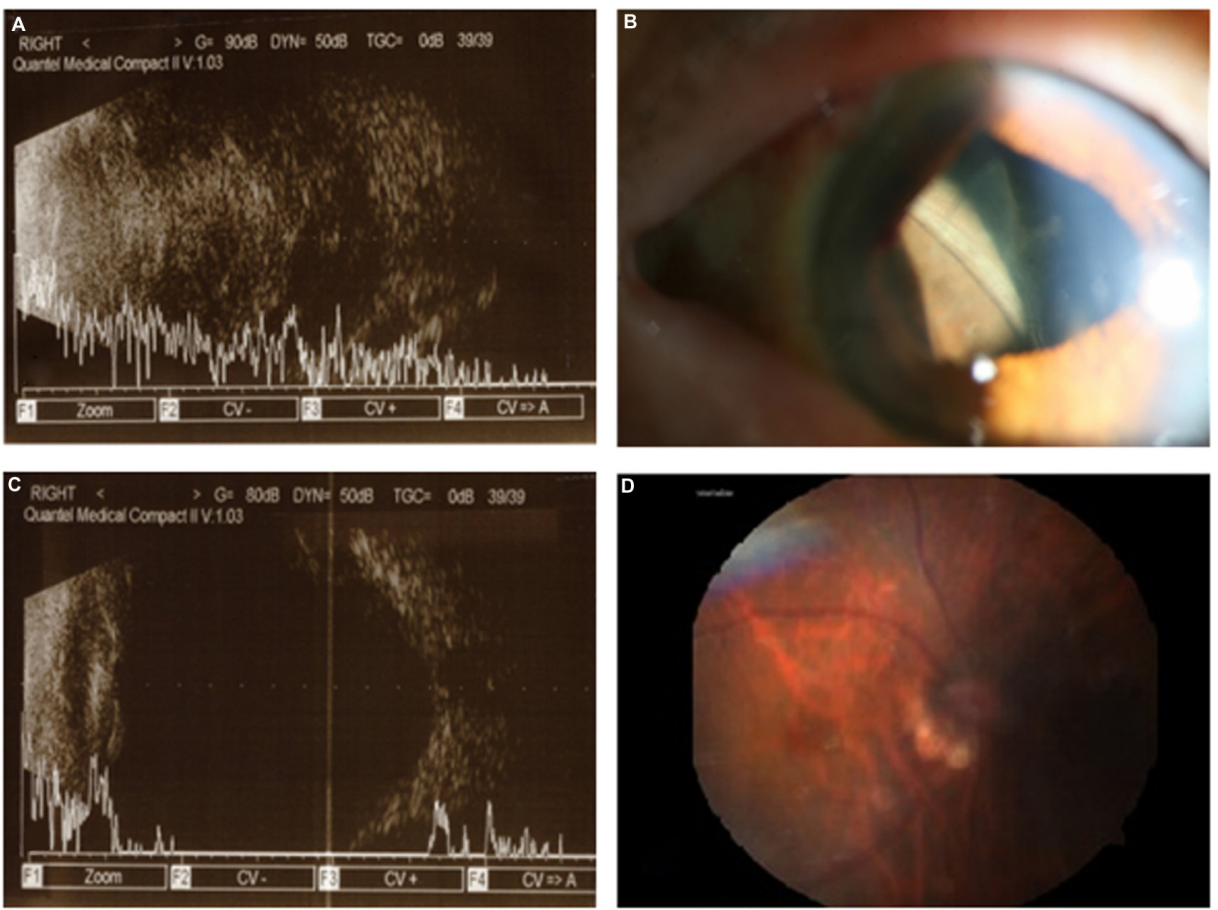

Figure I Changes in suprachoroidal hemorrhage with time.

Notes: (A) Ultrasonography at postoperative I day shows a massive suprachoroidal hemorrhage (SCH) with central retinal apposition. (B) Anterior segment photography at postoperative I day shows the SCH touching the intraocular lens. (C) Ultrasonography at postoperative 6 weeks reveals complete resolution of the SCH. (D) Fundus photography at postoperative 6 weeks depicts flat retina. 
more than 14 days was a risk factor of poor visual outcome, suggesting that surgical intervention may be necessary if the central apposition persists longer than 14 days. ${ }^{3}$ In our case, we decided to continue the conservative monitoring as resolution of central retinal apposition was found in 2 nd week, and the hemorrhage was completely absorbed in 6 weeks.

Although good initial visual recovery can be achieved with the resolution of the $\mathrm{SCH}$, previous studies revealed that long-term visual prognosis was poor because of subsequent retinal detachment or progression of underlying ocular disease. ${ }^{2}$ Therefore, this case is noteworthy, because good visual acuity of 20/60 with stable retina was maintained at 1 year after the expulsive $\mathrm{SCH}$.

Axial myopia increases the risk of $\mathrm{SCH}$ due to reduced scleral rigidity and choroidal vascular fragility. ${ }^{1,5}$ Surgical aphakia is also a predisposing factor of $\mathrm{SCH}$, because the loss of the lens and zonular support can promote stretching and separation of the uvea from the sclera during ciliochoroidal effusions. ${ }^{1}$ Advanced age and hypertension also associated with choroidal vascular fragility and sclerosis, and are considered to be risk factors of SCH. ${ }^{1,5}$ In the present case, the patient was 80 years of age, had hypertension and high myopia, all of which might contribute to the development of SCH. Moreover, aphakic state before sclera fixation might also aggravate the hemorrhage. Fortunately, the IOL fixed to sclera appears to prevent further protrusion of the choroid tissue. Early recognition followed by prompt meiosis and would closure might also prevent the deterioration of the $\mathrm{SCH}$. Factors associated with poor outcome, such as vitreous incarceration to the wound or retinal detachment, were never detected, which might also contribute to the visual recovery. ${ }^{2}$

\section{Conclusion}

This case report suggests that favorable anatomic and visual outcomes can be attained with conservative management even in cases of massive expulsive $\mathrm{SCH}$, particularly when central retinal apposition resolves in 2 weeks.

\section{Author contributions}

SJL carried out data acquisition and drafted the manuscript. JHL interpreted the data and drafted the manuscript. SWP interpreted the data and drafted the manuscript. MK analyzed the data and critically revised the manuscript. SBH analyzed the data and critically revised the manuscript. All authors read and approved the final paper, and agree to be accountable for all aspects of the work in ensuring that questions related to the accuracy or integrity of any part of the work are appropriately investigated and resolved.

\section{Disclosure}

The authors report no conflicts of interest in this work.

\section{References}

1. Chu TG, Green RL. Suprachoroidal hemorrhage. Surv Ophthalmol. 1999;43:471-486

2. Chu TG, Cano MR, Green RL, et al. Massive suprachoroidal hemorrhage with central retinal apposition. A clinical and echographic study. Arch Ophthalmol. 1991;109:1575-1581.

3. Scott IU, Flynn HW Jr, Schiffman J, et al. Visual acuity outcomes among patients with appositional suprachoroidal hemorrhage. Ophthalmology. 1997;104:2039-2046.

4. Meier P, Wiedemann P. Massive suprachoroidal hemorrhage: secondary treatment and outcome. Graefes Arch Clin Exp Ophthalmol. 2000;238: 28-32.

5. Chak M, Williamson TH. Spontaneous suprachoroidal haemorrhage associated with high myopia and aspirin. Eye. 2003;17:525-527.
International Medical Case Reports Journal

\section{Publish your work in this journal}

The International Medical Case Reports Journal is an international, peer-reviewed open-access journal publishing original case reports from all medical specialties. Previously unpublished medical posters are also accepted relating to any area of clinical or preclinical science. Submissions should not normally exceed 2,000 words or

\section{Dovepress}

4 published pages including figures, diagrams and references. The manuscript management system is completely online and includes a very quick and fair peer-review system, which is all easy to use. Visit

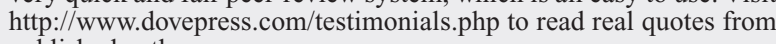
published authors. 